\title{
Erratum to: $17 \beta$-Estradiol-Induced Synaptic Rearrangements Are Accompanied by Altered Ectonucleotidase Activities in Male Rat Hippocampal Synaptosomes
}

\author{
Nataša Mitrović ${ }^{1}$ - Marina Zarić ${ }^{1}$ Dunja Drakulić ${ }^{1}$ - Jelena Martinović ${ }^{1}$. \\ Jean Sévigny $^{2,3} \cdot$ Miloš Stanojlović $^{1} \cdot$ Nadežda Nedeljković $^{4}$. Ivana Grković ${ }^{1}$
}

Published online: 29 December 2016

(C) Springer Science+Business Media New York 2016

Erratum to: J Mol Neurosci

DOI:10.1007/s12031-016-0877-6

The original version of this article unfortunately contained mistakes in Table 1 (the list of primary antibodies), referring to the host species of Abs.

The correct Table 1 is presented below:

The online version of the original article can be found at $\mathrm{http}: / / \mathrm{dx}$.doi. org/10.1007/s12031-016-0877-6.

Ivana Grković

istanojevic@vin.bg.ac.rs

1 Department of Molecular Biology and Endocrinology, VINČA Institute of Nuclear Sciences, University of Belgrade, Mike Petrovića Alasa 12-14, Belgrade 11001, Serbia

2 Département de microbiologie-infectiologie et d'immunologie, Faculté de Médecine, Université Laval, Québec, QC G1V 0A6, Canada

3 Centre de recherche du CHU de Québec, Université Laval, Québec, QC G1V 4G2, Canada

4 Institute for Physiology and Biochemistry, Faculty of Biology, University of Belgrade, Studentski trg 3, Belgrade 11000, Serbia 
Table 1 List of primary antibodies

Antibody specificity

Source, host species

Dilution (in TBST

or PBST)

Anti-phospho-p44/42 MAPK

(Erk1/2) (Thr202/Tyr204)

Anti-p44/42 MAPK (Erk1/2)

Anti-phospho-Akt (Ser473)

Anti-Akt

Anti-phospho-mTOR (Ser2448)

Anti-mTOR

Anti-syntaxin 1

Anti-synaptophysin

Anti-PSD95 clone 7E3-1B8

Anti-1

Anti-NCAM

Anti-Polysialic Acid-NCAM, clone 2-2B

Anti-NMDAR2B

Anti-NTPDase1/CD39

Anti-NTPDase3, KLH14

Anti-NTPDase2, BZ3-4F

Anti-CD73, rNu-9 $\mathrm{L}_{5}$

Anti- $\beta$-actin
Cell Signaling Technology (USA), rabbit polyclonal

$1: 1000$

Cell Signaling Technology (USA), rabbit polyclonal

$1: 1000$

Cell Signaling Technology (USA), rabbit polyclonal

$1: 1000$

Cell Signaling Technology (USA), rabbit polyclonal

$1: 1000$

Santa Cruz Biotechnology (USA), rabbit polyclonal

$1: 1000$

Santa Cruz Biotechnology (USA), mouse monoclonal

$1: 1000$

$1: 5000$

$1: 5000$

$1: 4000$

$1: 1000$

$1: 1000$

$1: 2000$

Merck Millipore (Germany), mouse monoclonal

$1: 1000$

Abcam (UK), mouse monoclonal

$1: 1000$

Abcam (UK), guinea pig polyclonal

$1: 1000$

T.L. Kirley, University of Cincinnati (USA), rabbit polyclonal (Belcher et al. 2006; Bjelobaba et al., 2010; Grkovic et al. 2016)

Université Laval (Canada), ectonucleotidases-ab.com, rabbit polyclonal (Sevigny et al. 2002)

Université Laval (Canada), ectonucleotidases-ab.com, rabbit polyclonal (Sevigny et al. 2002; Fausther et al. 2012; Mitrovic et al. 2016b)

Santa Cruz Biotechnology (USA), goat polyclonal
$1: 2000$

$1: 2000$

$1: 500$ 